\title{
Information as an Essential Component of the Biological Structures and Their Informational Organization
}

\section{Gaiseanu $\mathrm{F}^{*}$ \\ Principal Senior Researcher (Professor), Science and Technology of Information, Romania}

*Corresponding author: Florin Gaiseanu, PhD, Principal Senior Researcher (Professor), Science and Technology of Information Bucharest and Barcelona, Spain, Email: fgtext@yahoo.es

\section{Review Article \\ Volume 6 Issue 3}

Received Date: May 19, 2021

Published Date: July 01, 2021

DOI: $10.23880 /$ oajmb-16000198

\section{Abstract}

In this paper it is shown that information is involved in operations, independently of their source or support, chemical, physical or mathematical ones. Particularly, it is shown that info-structuration and info-destructuration are participatory processes typically in biological organisms, which can be represented schematically as a relation between the interacting components, highlighting that the info-structuration is an information-absorbing process and info-destructuration is an info-releasing one. Moreover, functions and functionality of an organism, as a combination of multiple interacting components, are actually expressed as information. It is also shown on this basis that can be distinguished and defined seven informational systems both in the human/sub-human bodies and in the living unit, which is the eukaryotic cell. An info-circuit of a sense perception can be described in terms of typical info-communication systems, as composed by information source (captured sensation), a coder (the involved sensor), a transmission channel (nervous system in animals or pathways in the cell), the decoder (brain in human and sub-human organisms or specialized reaction in the cell) and the displaying "screen" (prefrontal cortex in the brain or sensitive interpreter in the cell). Therefore, it is deduced that a proto-consciousness could be defined not only for the human and sub-human organisms, as rudimentary as the brain circuits are less complex in comparison with the brain, but also in the cell, marked by sentient-decisional pathways/micro-circuits and genetic transcription/translation (body info-(re) structuration) and/or replication (reproduction) processes.

Keywords: Information; Info-Integration/Releasing; Info-Structuration/Destructuration; Informational Systems And Circuits; Epigenetic Process; Consciousness/Proto-Consciousness; Cognitive Centers

Abbreviations: RMEs: Religious and Mystic Experiences; MIS: Maintenance Informational System; DNA: Deoxyribonucleic Acid; RNA: Ribonucleic Acid; GTS: Genetic Transmission System; IGG: Info-Genetic Generator; PIS: Programmed Informational Systems; IRSS: Informational Reactive Sentient System.

\section{Introduction}

Historically speaking, the concept of information has not been easy to be understood and this situation is still actual, at least as the implication of information in the functions and structuration of the biologic organisms is concerned [1]. The concept of information is used in general under common circumstances with the meaning of news or a communicated novelty. In a large sense this is true: when we speak about internet and the mass-media communication, the information unit expressed in Bits became something common, both as the consumed quantity of information and the info-transmission velocity are concerned. However, not too much information we associate to the specific processes regarding the participation of information to the structuring and functional abilities of the biological systems. Looking to the past, even since ancient times, Plato believed that the 


\section{Open Access Journal of Microbiology \& Biotechnology}

world, particularly the living world, is actually determined by "forms", which determine primarily the material reality, so the living matter is actually the "shadow" of these forms [2]. Translating this conception in our modern reality, this is equivalent to say that information ("forms") is actually the matrix of the world, specifically the living one. Moreover, thinking in modern concepts, we can observe for instance that the development of a seed to form the mature tree seems to follow a "software" internal program/algorithm to organize matter into the specific structure and functions [3]. Such a mysterious power is supported therefore by an a priori "knowing" of a well established "form", which drives the growth process according to the designed project for the living entity. The knowing itself is actually information, showing how to do this, according to a well established chain of operations, managing the small grains of matter composing the seed to follow a rigorous way of development. The printing machines of our nowadays are able to built various things, including a house, but always they are driven by a "knowledge", a "know-how" software, indicating the correct way of execution.

No too much progresses were registered on the attempts to interpret the living by means of information, that not only because of the complexity of the biologic "machines", but also because of the lack of profound understanding of the concept of information and its participation to their info-structuration/destructuration. Covering this lack, in a series of recently published studies dedicated to this issue, there were approached, discussed and advanced solutions to some still not understood problems, like the mind-body relation problem [2], the nature of mind and consciousness [3-5], the nature of life [6,7] and life cycle [8], the nature of attitude and behavior $[9,10]$ and the attitude evaluation $[11,12]$, the structure of consciousness $[13,14]$ and neuro-connections $[15,16]$, extra-sensorial phenomena [17], human as a bipolar info-matter structure [18], inherited talent or training alternative [19], certainty/ uncertainty in language communication [20], Religious and Mystic Experiences (RMEs) [14], Near Death Experiences (NDEs) [21] and immortality [22], optimization of a neurorehabilitation therapy [23]. As a continuation of this series, in this paper there are presented and discussed specific results showing how to apply the concept of information and infostructuration/destructuration to the biological systems and what informational systems compose, organize and manage such systems on the entire evolutionary scale.

\section{Information and Info-structuration/ Destructuration of the Biological Systems}

Although it has been the object of the human interest since unmemorable times [20], the question "What is life" remained in our collective consciousness and under the attention of various sciences like physics and chemistry [6], not only of the biology and philosophy $[7,2]$, especially after the famous Physicist Ervin Schrödinger addressed it to the scientific community [24]. This question sounds like a call to unite the efforts to unravel this mystery. Within this general effort, information science has not been involved too much on this realm, although according to the latest papers published recently, which shed a focused light on the contribution of information in understanding the properties and functioning of living systems, it was revealed the essential importance of information in creating, organizing and functioning of these systems [7]. If we regard this issue in a simple manner, the biologic systems could be perhaps compared with the programmed machines; however, these machines cannot feel, cannot decide and cannot reproduce themselves, so the question "what is life" is still remaining on the unsolved questions list of the humanity.

Among the attempts to introduce and understand the concept of information from the scientific point of view, one known approach is represented by the paradox of Maxwell's demon [25], which consists in a theoretical experiment in which a virtual "demon" would open a door between two containers with a gas in thermodynamic equilibrium, exactly when some molecules with higher velocity would attain it, so the gas in the second container would increase its temperature, violating in this way the second law of thermodynamics. However, in this experiment Maxwell did not taken into account that the intelligence invested by demon to measure the molecule velocity and to open at the suitable time the door is actually information. As it was shown later, the intelligence/knowledge of the demon can be expressed in terms of entropy, which must be counted to the maintenance of the entropic balance of this virtual process [26], so the second law of thermodynamics is not actually violated. On the other hand, this imaginary experiment, which was recently performed experimentally [27], shows that information can be converted in energy, leading to the increase of temperature in the second container up to $20 \%$.

Later, Draganescu stipulated from philosophic point of view that the living structures would be composed actually by matter and a matter species called "informatter", determining the specific properties [28]. We know now that the concept of "informatter" is actually information [4,5,13], which can be defined and measured in Bits in a binary (YES/ NO type) system in terms of probability as a certain event to be detected, in a communication system composed by a signal source and codifier, a transmission channel and a receiver provided with a signal decoder [29].

The composing parts of a biologic system communicate 


\section{Open Access Journal of Microbiology \& Biotechnology}

permanently. Moreover, some composing parts of the system are structured conveniently when it is necessary and other parts are decomposed consequently, the general homeostatic equilibrium maintaining the balance [30]. The understanding of the implication of information in a matter structuration/destructuration process is not so easy to be understood. For this, we have to take into account information as an operability agent in an interaction process, allowing to observe/measure the change in the configuration of a system composed by micro-components, determined by its entropy. Therefore, information is involved in an intervention, as a product of a factor of change, or a result of an operation or interaction, independently of the source of it, either from mathematical, chemical or physical processes. Any interaction is governed by laws, the structuring of any thing is also managed by interacting rules, which are actually informational substrate of such interaction. The universe itself is ultimately textured on an informational matrix of laws and rules, so information is actually a basic, fundamental component not only of the living systems-the biologic world, but also of our material universe of non-living matter [3]. The functionality of a system, particularly that of the biologic organisms, is actually intrinsically carried out by informational chains, orienting the system to a goal or an objective, either proposed/predictable or not. Functionality of a system is actually traduced as information.

To define the info-structuration process, let's consider a simple system of two components. Therefore, if A and B are the components of a system under interaction determined by a law/rule of this interaction/operation assisted by information $I$, this process can be represented by the following general basic relation:

$$
(\mathrm{A}+\mathrm{B})+\mathrm{I} \Leftrightarrow \mathrm{AB}(\mathrm{I})
$$

Where (I) represents the intrinsic (incorporated) "embodied" information [13], reflecting the hided, but still preserved operability of information as an active agent within the new $(A B(I))$ structure. In rel. (1) the balance to the right side corresponds to a structuration process (inherently with absorption of information), while the balance to the left side a destructuration process, with release of "free", virtual information.

The quantity of information I can be calculated as a difference of entropy $\mathrm{H}$ of a multi-structured system with $\mathrm{N}$ components, which changes its configuration between an initial and a final state, by the elimination of a quantity of incertitude $\Delta H$, i.e.:

$$
\mathrm{I}=\Delta \mathrm{H}=\log _{2} \mathrm{~N}
$$

when all components dispose of the same probability of operation. If $\mathrm{N}=2$, it is obtained the Bit informational unit. Rel. (2) is the simplest form for calculation, but for concrete cases of configuration, the calculations are much more complex.

On the other hand, while a destructuration corresponds usually with naturally increased entropy, the structuration corresponds with absorption of information. A specific example of natural destructuration is the diffusion process of an assemble of particles into a liquid or solid material [1], showing that the particles are spread from high to low concentration. The biologic systems work permanently to decrease their entropy, opposing themselves therefore to the natural tendency dictated by the second thermodynamic law which requires the natural increase of entropy. This is obtained actually by absorption of information for structuration/restructuration [6,7] and the elimination of heat in the external environment, like a refrigerator machine.

From the informational point of view, in biologic systems it is therefore important to distinguish information in two main states: (i) matter-related information, described by the forward sense of rel.(1) [7,2,31], and (ii) virtual/free/released information described by the revers process in rel. (1), ready to be included into another chain of specific interactions/ algorithms, with specific functions. When we refer to the operability of mind, we have to observe that this works with concepts and informational criteria (beliefs, rules). In the cell, the unit of the biologic systems, the warehouse of infostructuration is the molecule of deoxyribonucleic acid (DNA) of genes, and the main informational messenger/transporter is the ribonucleic acid (RNA).

\section{Informational Organization of the Human and Sub-Human Organisms}

The compartmentalization (specialization by organs) is one of the biological strategies to decrease the entropy by info-structuration on the evolution scale. The informational management of the human and sub-human body is centralized and supported by the brain, which is hieratically organized, reflecting the info-development on the evolution scale, from the basic functions and structures of the brain stem to the complex functions and structures of the cortex [15], as it is represented by the info-evolution blue arrow in the right upper side in Figure 1. The integration of the external cue/ stress environment information is performed by epigenetic processes [16,32], as it is represented by the info-integration pink arrow in the left upper side of Figure 1. 


\section{Open Access Journal of Microbiology \& Biotechnology}

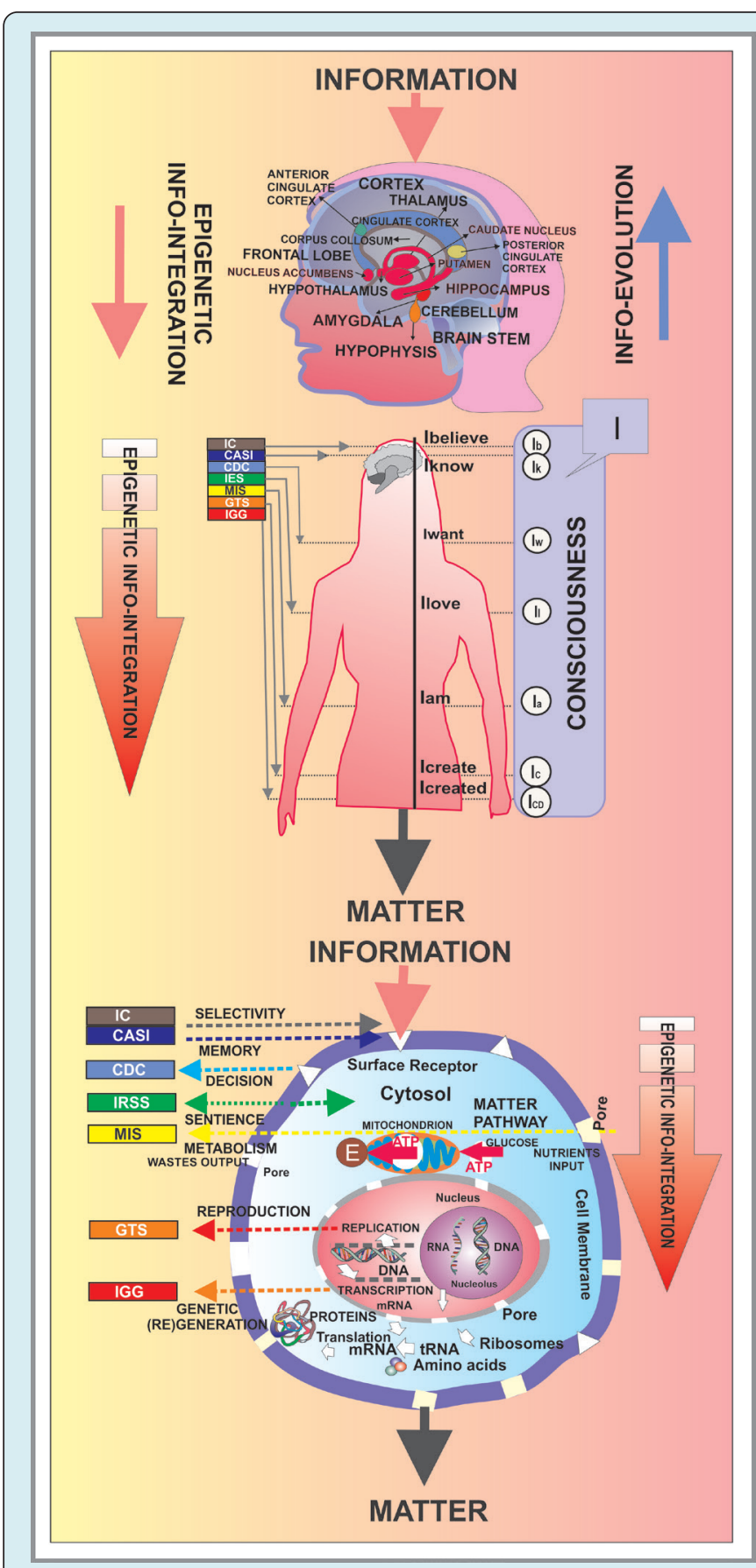

Figure 1: Schematic representation of the informational system of the human body and of the eukaryotic cell.

The epigenetic processes are examples of the reactive/ adaptive response of the organisms to an insistent (intensive and/or repetitive) external input information, finally ended by a stable info-structuration process as indicated by rel. (1) heading toward the right side, which determines the modification of the genetic system, but without the change of the info-genetic configuration specific to the species. The epigenetic process includes three phases [32]: (1) in the first stage, the initial stress signal-the epigenetor, interacts by a chain of reactions with the body cell, sensitive to the external environmental changes; (2) in a second phase, the epigenetor triggers the initiator, as a repetitive chain-type signal sufficiently intensive to initiate the epigenetic process itself; (3) in a third phase, the maintainer is the informational signal which preserves the changes in the DNA structures, manifested by acquired new traits, which can be transgenerationally transmitted. Such a process could therefore explain the evolution.

According to the above discussion, the analysis from informational point of view of the specific functions managed by the brain, allows to define the following informational components [13,15], described as below and represented in the upper, central and left side of Figure 1. CASI is the center of acquiring and storing of information, connected to external (sight, hearing, smell, taste, touch) and internal (hungry, thirst, pain) sensors of information and mainly with prefrontal cortex (short-term memory) and hippocampus (long-time memory).

CDC is the center of decision and command, connected to CASI to activate the necessary information for info-operation according to the circumstances, to make a decision and to transmit it to the execution elements (muscles), particularly to the vocal system, and is connected especially to the cortex hemispheres of the brain. IES is the info-emotional system managing the emotions as a response to the received and memorized information and is connected with the limbic system composed by amygdala, hippocampus, thalamus, hypothalamus, and surrounding zone (caudate nucleus, putamen, and nucleus accumbes) and with the heart zone to control the blood administration according to the emotional necessities.

MIS is the maintenance informational system managing the metabolic processes of foods, water, air, which provides energy and reconstruction material of the body, connected to brain stem as a relay between cerebrum, cerebellum and spinal cord and medulla and mainly with the digestion apparatus in the body. GTS is the genetic transmission system, connected to hypophysis and hypothalamus in the brain and with reproduction apparatus in the body. IGG is the info-genetic generator, which manages the body development according to the age, and is connected to hypophysis, hypothalamus and basal ganglia.

IC is the info-connection pole connected to the anterior cingulate cortex, which controls the YES/NO type selective transmission of information to the frontal cortex of conscious judgment area. As it was recently shown, this is involved in the Religious and Mystic Experiences (RMEs) [14], the extra-sensorial phenomena [17], particularly in the 


\section{Open Access Journal of Microbiology \& Biotechnology}

extra-corporal detection of reality during NDEs experiences [21] and even in extra-consciousness duration after the body death [22]. The posterior cingulate cortex is involved in the switching of attention from the external reality to the internal world, during a day-dreaming process [33]. The epigenetic processes are initiated therefore in the inforeception zones in CASI, following a running informational mechanism toward the right side of rel. (1) with absorption of information. If the received stress-message is important and/or intensive/repetitive, this is transferred from short to long-time memory, analyzed by $\mathrm{CDC}$, together with the participation of IES, converted in an automatic skill by MIS and finally integrated by epigenetic mechanisms in the genes of the body cells. Therefore, the surrounding information can be absorbed/integrated/"embodied" into the genetic system in stable structures, by the reconfiguration of DNA genes, without affecting however the species characteristics, as it is suggestively represented by the wide arrow in the left central side of Figure 1. This new acquired trait is manifested by the corresponding gene expression during the transcription/translation process of the gene information for specific protein formation, the building blokes of the body, and can be transmitted to the next generations. From reasons of efficiency/economic operability of this process patented by nature for integration of information, we have to suppose that partial epigenetic mechanisms (1-st, 2-nd stage), which can be described by rel. (1) (forward/reverse sense) are also involved in both short and long-term memory processes for info-integration/recall by means of the thought as informational operator.

The projections of the activities of the informational systems described above (CASI, CDC, IES, MIS, GTS, IGG, IC) => (I know, I want, I love, I am, I create, I created, I believe) are defined as cognition centers of consciousness, specifically called as following: I know is the memory, accumulating the entire life experience from reality and connected to the informational input; I want is the decision center representing the output of information (attitude); I am expresses the own power, health, vigor and self-status; I create is the cognitive center of creation (of a new generation), representing also the info-genetic output; I created (by parents) is the info-genetic input of the inherited characteristics, I believe representing the selection criteria and beliefs, as schematically shown in the central side of Figure 1.

We can understand the processing of the received signals in terms of communication within specific circuits as follows: referring for instance to consciousness in informational terms, but reduced only to the visual aware, the input information source would be the captured image itself, coded then by sensors in a specific electrical/chemical way, transmitted by the nervous system, which plays the role of a transmission channel, the received signal is decoded then by the brain and the final information is received/projected/ displayed on the prefrontal "screen", like in the electronic communication systems. In the same way can be described the corresponding circuits of other informational signals communicating information from the external and internal sources. According to the above discussion, we have to observe [33] that when an image is recalled from memory, the same visual circuits and prefrontal screen are used. Admitting that the coding/decoding process is performed by means of a partial epigenetic mechanism based on incorporation/ release of information as described by rel. (1) running to the right/left side, we have the picture of the operating infomechanisms, not understood up to date. Actually, we have to regards the memory as a general informational store with various degrees of integration, as described above: shortterm and long-term memory amplified by emotions and/ or decisions by implication and/or repetition, info-abilities, epigenetics. This is actually a reversible mechanism, in the sense that the information can be recalled by using the same operational circuits. The repetition is a key of the maintenance of information integrated in the system, as stable as possible. If not, the information is gradually lost, including the new epigenetic traits.

CDC is an info-operational system based on decisions criteria and commands to the execution elements, with access to CASI, from where recalls the necessary information by the thought, which is the informational operator, and where stores the decision. The signals from IES, MIS, GTS and IGG are also stored in memory, so CASI is actually a central and characteristic center of life [2]. While CASI, CDC and IES allow the adaptation acceding to the external and internal circumstances by means of the so defined OIS=CASI+CDC+IES (operative informational system), MIS, GTS and IGG are automatic/autonomic programmed informational system (PIS=MIS+GTS+IGG) for body/life maintenance and longterm adaptation by genetic/epigenetic processes.

According to the brain evolutionary process induced by information [34] and to the informational structure of the human body as presented above, we have to deduce that the sub-human organisms dispose also of consciousness, as more rudimentary as the brain disposes of less complexes circuits, compared with the human ones.

\section{Informational Organization of the Eukaryotic Cell Organisms}

The eukaryotic cell is the life unit mainly for animals, plants and most of algae, but can be either single or as a part of the multicellular organism (Figure 1 bottom side). Therefore, the eukaryotic cell is an auto-organized and autonomic unit of life which can live alone, distinct from the prokaryotic cells (mainly bacteria), which has not organelles 


\section{Open Access Journal of Microbiology \& Biotechnology}

well defined into the cytoplasm. Although not evident at a first glance, the functions of the eukaryotic cells are equivalent with that of the multicellular organisms, particularly of the animals $[6,7,35]$. The cell outer membrane is equivalent with the skin, the Golgi apparatus with the heart, distributing the nutrients and oxygen to the body, connected with the rough/smooth endoplasmic reticulum, which is equivalent to the pancreas/blood vessels functions in animals. This is involved in protein synthesis like insulin (produced in the pancreas) and cholesterol (used in membrane formation) and their folding. The role of the endoplasmic reticulum in the animal organisms refers also to the detoxification of harmful drugs and of metabolic by-products in liver, whereas in the reproductive organs, smooth endoplasmic reticulum produces the steroid hormones testosterone and estrogen. The vacuoles are the "stomach" of cells, storing food and water for further use, the ribosomes and mitochondria (with membranes specialized for aerobic respiration) corresponds with bowels and lungs because they produce/extract proteins and oxygen-assisting energy (adenosine triphosphate - ATP) from foods. The cytoplasm is a dynamic support structure of organelles in the cell like muscles, while cytoskeleton the static resistance structure, like bones in the body.

The endoplasmic reticulum (with multi-functional role), mitochondria (energetic aerobic-oxygen-assisted resource), Golgi apparatus (transporter/distributer), lysosomes (equivalent of the spleen in animals for degradation of wastes/obsoleted products), peroxisomes (liver-kidney detoxifying role) [36], vacuoles (stomach-like reservoirs), ribosomes (intestines) operating as organelles with specific digestive/metabolic functions in the eukaryotic cells, are parts of a maintenance informational circuit of the cell. As the metabolic processes are automatically driven on such specific pathways, we can define a maintenance informational system (MIS) of the cell.

The informational controlling master of the cell is the nucleus, which stores/holds the entire informational memory of the cell, both as the info-structuration and traits is concerned, seemingly similar with the centralized informational management operated by the brain. The multiplication of the cell is driven during the replication process allowing the reproduction, so a genetic transmission system (GTS) of the cell can be also defined. Such a process is assisted by RNA and enzymes, starting with the split (destructuration) of the two DNA strands and the restructuration into the mother and daughter DNA structures. The info-genetic generation process is represented by the transcription (the copy of the sequences of gene organization driven by messenger mRNA plus enzymes) and the translation mechanism into specific proteins, with the participation of amino acids and transfer tRNA from ribosomes. Therefore, a info-genetic generator (IGG) system could be also defined, like in the human organism, allowing the development/ (re)construction/(re)generation of the cell during its life cycle. Like in the human body, MIS, GTS and IGG represents programmed informational systems (PIS), indispensable for the maintenance of life and long-time survival. The long-time adaptation process to the surrounding cues/stress is assured by an info-integration epigenetic process at the cellular level, as was already described above and represented suggestively by the bottom right side arrow in Figure 1.

The semitransparent membrane of the cell, the precursor of the human skin, plays a fundamental role for a selective YES/NO-type binary mechanism of the transfer from/to the cell of the necessary nutrients/wastes and informational agents of matter/energy/information interchange with the environment. This selective process is permitted by specific local "devices" on the cell membrane, represented by receptors, as it is shown in the bottom side of the Figure 1. The receptors are basically proteins which allow the binding of chemical signal external agents only if these are complementary structured compared with the receptor' specific configuration. This property is actually interpretable as a local memory, because only the previously proved fitted information can be received. Moreover, as by epigenetic mechanisms/pathways/micro-circuits new traits can be acquired for adaptation, this memory is extended during the life cycle of cell with new info-type receptors, created by means of transcription/translation models. The network of receptor memory, together with corresponding differential chemical reactive pathways/micro-circuits of decision, up to epigenetic acquired (stable) traits, represents a combined CASI + CDC operative system of the cell, ready and operating to learn for adaptation. This is actually a fundamental property of the biological systems, supporting the survival effort under distinct and variable surrounding conditions, where the finding of foods is one of the primary necessities.

A new intervening factor/signal in the physical-chemical panorama of the steady-state regime of the cell, will be "felt" by the interpretation sensors in distinct/differential ways as a "emotions" or "drug"-type induced emo-states. For reasons of survival, the most important probably "feeling" sensation interpreted by the cell as a response/reaction of such outside information are the danger signals, manifested at the top of the evolution scale in human by the amygdala operability in the limbic system, part of IES. By contrary, under a steadystate regular regime, this situation can be differentially detected/"felt"/interpreted as a peace/harmony/"love" state by the feeling detection system, like in the human body organism. This is an equilibrium homeostatic reference line [30] between YES/NO acceptable/rejectable felt-like/ interpreted signals, according to own and/or ancestral 


\section{Open Access Journal of Microbiology \& Biotechnology}

(genetic) experience accumulated into the memory system of the cell, so an informational reactive sentient system (IRSS) of the cell, as a generalization of IES in human can be defined.

Therefore, we can speak about an existent protoconsciousness (although rudimentary), on surrounding and inner events, represented by OIS=CASI+CDC+IRSS, at least as the decisional info-operative-sentient perception is concerned, working by an informational YES/NO binarytype decisional process. As the transcription/translation (IGG) and replication (GTS) type processes are involved deeply in the structuration/restructuration of the cell, these should be "felt" also as major/crucial events in the life cycle, contributing to the proto-consciousness operability of the cell. On the evolutionary scale, we may suggest that this was developed gradually under various forms till the top-kind OIS informational system of the human body. Moreover, the extra-sensitive experience of the cell, corresponding to IC pole of the human informational system, could be understood for instance by the ability of bacteria, which are able to communicate synchronously with other members, in order to form geometrically organized colonies [6], or at birds by geographical orientation through extra-sensorial signals assisted by quantum processes for migration, and at some animals and fishes, by sensitivity to detect in advance some coming natural cataclysms or bad weather.

From the above presentation it can be observed that the biologic organisms are actually connected to information and matter, one of them - information, necessary to adaptation and survival, and another one - matter, for maintenance of the body structure and long-term survival of the species, as it is suggestively represented in Figure 1. The biological systems are therefore binary info-material dynamic self-organized structures, maintaining their existence by adaptation and learning processes. The remarkable advances in the understanding of the info-organization and functionality of the biologic organisms meet the parallel advances in the artificial intelligence referred to learning operability [37] and memory [38], to biomedical devices like silicon capacitive sensors for biomedical applications [39-41], biocompatible devices like bio-computers and bio-transistors with DNA and RNA, biological memories, molecular imaging DNA-based cripto-systems, genetic neuronal relays, bi-dimensional DNA nanostructures, FET bio-transistors with carbon nanotubes [31], allowing a close inter-correlation for stimulating mutual development in the field of health diagnostic and treatment.

\section{Conclusion}

It was shown that the information is related to operation, perceived as a new event district from the rest, or as certitude, measurable in a binary YES/NO system by probabilistic methods as a difference between the entropy in two distinct states. Info-structuration/destructuration is an indispensable concept to describe the activity/functionality of the biological systems, as a consequence of the interaction between their micro-components, independently of the source, either from mathematical, chemical or physical processes and can be suggestively understood by the incorporation/integration/"embodiment' of information in the new structure and the release as a "free"/"disembodied" information, ready to activate further structuration chemical chain processes.

A typical process involving cascade of chemical interactions is the epigenetic process, which consists in the absorption/integration of information from surroundings cue/stress to the structure of DNA in the body, without affecting the typical structure of the species, as a reactive/ adaptive response of the organisms to an insistent (intensive and/or repetitive) external input information. Taking into account the body interaction with information, it was shown that the functionality of the human and sub-human organism is related by seven informational distinct components of the informational system, referring to the memory, decision, emotions, self-status, genetic transmission, genetic inheritance and info-selectivity, both as informational circuits including specific managing regions of the brain and body organs as transducers and/or execution elements, and as a projection in consciousness by cognitive centers. In terms of information, consciousness is actually the consequence of the decoding of information operated by the brain and projected/displayed on the prefrontal mind "screen" if only the image circuit is considered, as the end of a communication system formed by input information, a coder (sensor), communication channel (nervous system), receiver (brain) and info-decoder. According to the presented discussion, the sub-human organisms dispose also of consciousness, as rudimentary as the brain disposes of fewer complexes circuits, compared with the human ones.

It was deduced that a rudimentary proto-consciousness on the surrounding and inner events at least as the decisional info-operative-sentient perception of the cell is concerned, on which the replication process should have a major impact, working by an informational YES/NO binary-type process with decisions and decision criteria. The detailed analysis of the organelles of the eukaryotic cells and the comparison with the functionality of the human and sub-human organisms shown that practically on the entire evolutionary scale of organisms the informational organization is the same, with various degrees of evidence. The results analyzed in this paper become a basis for further development addressed to biological structures, to artificial informational and biocompatible systems for mutual benefice. 


\section{Open Access Journal of Microbiology \& Biotechnology}

\section{Acknowledgment}

To Academician Florin Filip and Gheorghe Stefan for stimulating discussions and support during the presentation at the Romanian Academy on March $18^{\text {th }} 2021$, and to all the participants at that meeting, to the Science and Technology of Information in the Romanian Academy.

To Adrian and Ana-Maria Gaiseanu for permanent support.

\section{References}

1. Gaiseanu F (2021) Informational Model of Consciousness and Life, Information as a Constitutive Element of the Living Systems: from Philosophy to Modeling and Applications. Colocviile Mihai Draganescu, Presentation at Romanian Academy, Science and Technology of Information, on March $18^{\text {th }}$.

2. Gaiseanu F (2021) Solution to the mind-body relation problem: Information. Philosophy study 11(1): 42-55.

3. Gaiseanu F (2016) Consciousness as Informational System of the Human Body. Consciousness and Life Physics. Cosmology and Astrophysics Journal 16(1): 1425.

4. Gaiseanu F (2018) Information: From Philosophic to Physics Concepts for Informational Modeling of Consciousness. Philosophy Study 8(8): 368-382.

5. Gaiseanu F (2019) Informational Model of Consciousness: From Philosophic Concepts to an Information Science of Consciousness. Philosophy Study 9(4): 181-196.

6. Gaiseanu F (2020) What Is Life: an informational model of the living structures. Biochemistry and Molecular Biology 5(2): 18-28.

7. Gaiseanu F (2020) Informational structure of the living systems: From philosophy to informational modeling. Philosophy Study 10(12): 795-806.

8. Gaiseanu F (2019) Destiny or Free Will Decision? A Life overview from the perspective of an informational modeling of consciousness Part I: Information, Consciousness and Life Cycle. Gerontology and Geriatric Studies 4(3): 1-6.

9. Gaiseanu F (2018) Destiny or Free Will Decision? A Life overview from the perspective of an informational modeling of consciousness Part II: Attitude and Decision Criteria, Free will and Destiny. Gerontology and Geriatric Studies 4(1):1-7.
10. Gaiseanu F (2020) Attitude as an expressible infooperational reaction to a perceived/purposed Object/ Objective. International Journal on Neuropsychology and Behavioral Sciences 1(1): 12-16.

11. Gaiseanu F (2021) Evaluating attitude and behavior: An info-operational procedure Related/Supported by the cognitive centers of mind. International Journal on Neuropsychology and Behavioural Sciences 2(1): 1-5.

12. Gaiseanu F (2021) Mental aggressive operability from informational perspective: A deterrence Manifesto. EC Neurology 13(4): 31-39.

13. Gaiseanu F (2019) The Informational Model of Consciousness: Mechanisms of Embodiment/ Disembodiment of Information. Neuro Quantology 17(4): 1-17.

14. Gaiseanu F (2019) Human/Humanity, Consciousness and Universe: Informational Relation, Neuro Quantology 17(5): 20-30.

15. Gaiseanu F (2019) Informational Neuro-connections of the Brain with the body supporting the informational model of consciousness. Archives in Neurology and Neuroscience 4(1): 1-6.

16. Gaiseanu F (2019) Informational Mode of the brain operation and consciousness as an informational related system. Archives in Biomedical Engineering and Biotechnology 1(5): 1-7.

17. Gaiseanu F (2017) An Information based model of consciousness fully explaining the mind Normal/ Paranormal Properties. Neuro Quantology 15(2): 32-40.

18. Gaiseanu F (2020) Information-matter bipolarity of the human organism and its fundamental circuits: from philosophy to physics/neurosciences-based modeling. Philosophy Study 10(2): 107-118.

19. Gaiseanu F (2019) The silent voice of those who are no longer: Transgenerational Transmission of Information from the perspective of the informational model of consciousness. Gerontology \& Geriatric Studies 5(1): 482-487.

20. Gaiseanu F (2019) Language patterns and cognitivesentient reality: certainty/uncertainty in cognitivesentient exploration of reality. Media models to foster collective human coherence in the psyc hecology, Stephen Brock Schafer (Ed.), IGI Global, USA, pp: 49-72.

21. Gaiseanu F (2017) Quantum-assisted process of disembody under near-death conditions: An Informational-Field Support Model. Neuro Quantology 


\section{Open Access Journal of Microbiology \& Biotechnology}

15(1): 4-9.

22. Gaiseanu F (2018) Near-death experiences and Immortality from the perspective of an informational modeling of consciousness. Gerontology \& Geriatric Studies 2(3): 1-3.

23. Gaiseanu F (2020) Multitask music-based therapy optimization in aging neurorehability by activation of the informational cognitive centers of consciousness. Gerontology \& Geriatric Studies 6(3): 1-5.

24. Schrödinger E (1944) What is life? The physical aspect of the living cell. Cambridge University Press, Cambridge, UK.

25. Dougal RC (2015) Kelvin, Maxwell, Clausius and Tait: the correspondence of James Clerk Maxwell. WIT Transactions on State of the Art in Science and Engineering 89: 135-151.

26. Szilard L (1964) On the decrease of entropy in a thermodynamic state by the intervention of intelligent beings. Behav Sci 9(4): 301-310.

27. Toyabe S, Sagawa T, Ueda M, Muneyuki E, Sano M (2010) Experimental demonstration of information-to-energy conversion and validation of the generalized Jarzynski equality. Nature Physics 6: 988-992.

28. Draganescu M (1990) Informatia materiei (Information of matter), Editura Academiei Române, București.

29. Shannon CE (1948) A mathematical theory of communication. Bell Syst Tech J 27: 379-423.

30. Gaiseanu F (2020) Informationally-assisted equilibrium and health: specific acc contribution from the perspective of the informational model of consciousness. EC Psychology and Psychiatry J 9(5): 37-49.

31. Gaiseanu F (2020) Fizica Constiintei si a Vietii: Modelul Informational al Constiintei-Informatia in Neurostiinte, Biocomputeresi Biosisteme (Romanian version); (Physics of Consciousness and Life: Informational Model of Consciousness-Information in Neurosciences, Biocomputers and Biosystems) Globe Edit (Omni Scriptum International Group): Forewords by M. Pregnolato, S. Schafer and D. K. J. Meijer. Closure Endorsement Words: D. Radin and A. A. Attanasio, pp: 1-341.
32. Gaiseanu F (2019) Epigenetic Information-body interaction and information-assisted evolution from the perspective of the informational model of consciousness. Archives in Biomedical Engineering and Biotechnology 2(2): 1-6.

33. Gaiseanu F (2020) Info-Relational cognitive operability of the posterior cingulate cortex according to the informational model of consciousness. International Journal of Psychological and Brain Sciences 5(4): 61-68.

34. Gaiseanu F (2020) Information based hierarchical brain organization/evolution from the perspective of the informational model of consciousness. Archives in Neurology \& Neuroscience 7(5): 1-9.

35. Almoney K (2015) The Cell Compared with the Human Body.

36. Lodish H (2000) Molecular Cell Biology. $4^{\text {th }}$ (Edn), New York: W.H. Freeman.

37. Filip F (2020) DSS-A class of evolving information systems in Dzemyda G, et al. (Eds), Data science: New issue, challenges and applications, Springer, pp: 253277.

38. Stefan G (1998) The Connex memory: a physical support for tree/list processing. Romanian Journal of Information Science and Technology 1(1): 85-104.

39. Gaiseanu F, Postolache C, Dascalu D, Esteve J, Tsoukalas D, et al. (1998) Material and device characterization for process optimization of the capacitive pressure sensors for biomedical application. Romanian Journal of Information Science and Technology (Romanian Academy) 1(1): 85-104.

40. Gaiseanu F (2013) Contributions to the modeling and simulation of the atomic transport processes in silicon and polysilicon and applications. Proceedings of the Romanian academy, Series A, The Publishing House of the Romanian Academy 4(4): 376-384.

41. Gaiseanu F (2017) Modeling and Simulation of the Impurity Diffusion and Related Phenomena in Silicon and Polysilicon Systems in Microfabrication and Micromachining Technologies. Annals of the Academy of Romanian Scientists. Series on Science and Technology of Information 10(1): 41-78. 